\title{
A Novel Long-Term ex vivo Model for Studying Vascular Calcification Pathogenesis: The Rat Isolated-Perfused Aorta
}

\author{
Mirjam Schuchardt ${ }^{a} \quad$ Nathalie Vanessa Siegel ${ }^{a} \quad$ Milen Babic $^{a}$ \\ Alexander Reshetnik $^{a}$ Ronald Lützenberg ${ }^{b}$ Walter Zidek ${ }^{a}$ \\ Markus van der Giet ${ }^{a} \quad$ Markus Tölle $^{a}$ \\ ${ }^{a}$ Charité - Universitätsmedizin Berlin, corporate member of Freie Universität Berlin, Humboldt Universität zu Berlin, \\ and Department of Nephrology, Berlin Institute of Health, Berlin, Germany; ${ }^{\mathrm{b}}$ Klinik für Plastische, Ästhetische und \\ Handchirurgie, Universitätsklinikum Magdeburg, Magdeburg, Germany
}

\section{Keywords}

Aortic perfusion - Ex vivo mineralization · Smooth muscle cell · Vascular calcification

\section{Abstract}

The investigation of vascular calcification and its underlying cellular and molecular pathways is of great interest in current research efforts. Therefore, suitable assays are needed to allow examination of the complex calcification process under controlled conditions. The current study describes a new ex vivo model of isolated-perfused rat aortic tissue with subsequent quantification and vessel staining to analyze the calcium content of the aortic wall. A rat aorta was perfused ex vivo with control and calcification media for 14 days, respectively. The calcification medium was luminally perfused and induced a significant increase in calcium deposition within the media of the vessel wall detected alongside the elastic laminae. Perfusion with control medium induced no calcification. In addition, the mRNA expression of the osteogenic marker bone morphogenetic protein 2 (BMP-2) increased in aortic tissue after perfusion, while SM22a as smooth muscle marker decreased. This newly developed ex vivo model of isolated-perfused rat aorta is suitable for vas- cular calcification studies testing inducers and inhibitors of vessel calcification and studying signaling pathways within calcification progression.

\section{Introduction}

Calcification of the vascular wall remains a major cause of morbidity and mortality in elderly patients as well as in patients with a high cardiovascular risk, e.g., those with end-stage renal disease [1]. However, a specific therapy treating vascular calcification is currently not available [1]. Considerable efforts within this research field have provided more insight into the pathogenesis, contributing factors, and associated cellular mechanisms [2-4].

Different experimental settings, i.e., in vitro assays using vascular smooth muscle cells (VSMC), ex vivo assays using aortic rings, and in vivo animal models mainly with rodents, have been established for studying the complex processes of vascular calcification. Each model differs in complexity and has assets and drawbacks. In vitro cell experiments lack the complex environment of the vessel ar-

\section{KARGER}

(c) 2019 S. Karger AG, Basel

E-Mail karger@karger.com

www.karger.com/jvr
Prof. Dr. Markus van der Giet

Department of Nephrology, Charité - Universtitätsmedizin Berlin

Hindenburgdamm 30

DE-12203 Berlin (Germany)

E-Mail markus.vandergiet@ charite.de 


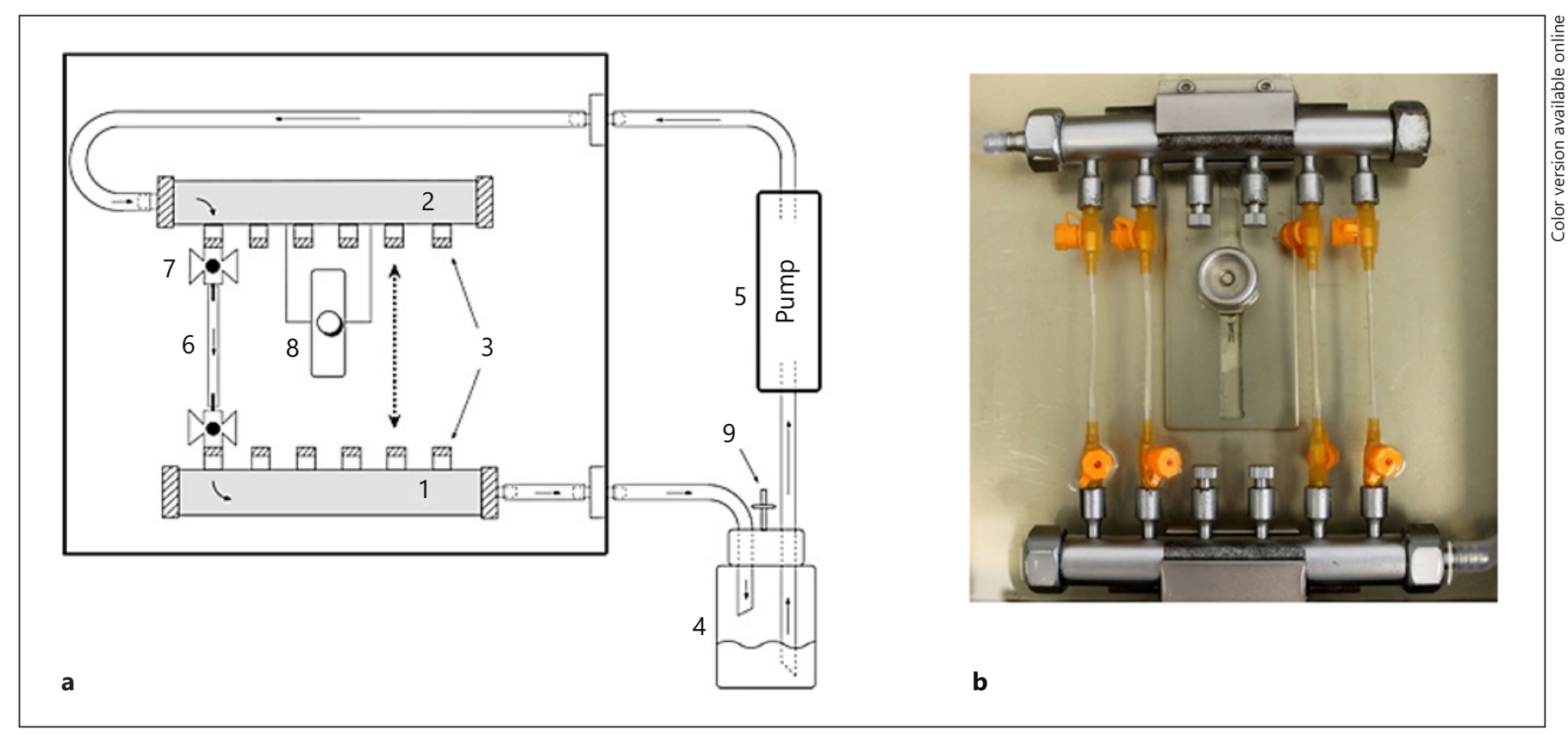

Fig. 1. Perfusion chamber scheme. a Perfusion chamber diagram. 1: fixed module, 2: mobile module, 3: aortic clamping, 4: perfusion media tank, 5: pump, 6: aorta, 7: catheter, 8: movable part, and 9: filter for gas exchange. The chamber is closed with a lid includ- ing 4 filters for gas exchange. The chamber consists of stainless steel. All chamber and tubing parts are autoclavable or used as sterile disposables. b Snap of the chamber with 4 aortas between the fixed and mobile modules. chitecture. Ex vivo experiments with aortic rings lead to stimulation via both the internal and the external vessel surfaces. Therefore, it is not possible to distinguish between these variable influences on vascular calcification. In vivo models of different diseases in knockout or transgenic animals could help to identify the influence of one specific target molecule. For screening experiments and treatment studies, in vivo models are time-consuming, expensive, and associated with suffering of animals. Therefore, the development of a novel experimental ex vivo setting is necessary to simulate the vascular calcification process by the perfusion technique exclusively via endoluminal stimulation.

This methodological paper describes a new ex vivo method of a rat isolated-perfused aorta (IPA) for 14 days under sterile humidified conditions at $37^{\circ} \mathrm{C}$ with subsequent analysis of vessel calcification by calcium quantification and histological examination. In addition to a protocol description, results of induced vascular calcification are shown as proof of concept. These ex vivo model might bridge the experimental gap between the current available ex vivo and in vivo methods for vascular calcification experiments.

\section{Materials and Methods}

\section{Materials}

Buffer and cell culture media were purchased from Biochrom (Berlin, Germany). Salts and staining solutions were purchased from Carl Roth (Karlsruhe, Germany) and Sigma-Aldrich (Steinheim, Germany).

\section{Animals}

Animals were handled in accordance with the guidelines of directive 2010/63/EU of the European Parliament on the protection of animals used for scientific purposes and the Animal Committee of Charité Berlin, Germany. Male Wistar rats were purchased from Janvier (Le-Genest-Sain-Isle, France). The animals were fed a normal chow diet with water ad libitum. They had a day-night cycle of $12 \mathrm{~h}$ in a humidified atmosphere. Prior to the experiments, there was at least 1 week for acclimatization. All of the animals were weighted to ensure accurate drug dosage calculation for anesthesia. The rats used for the experiments were 2 months old (median age).

\section{Experimental Setting}

The perfusion chamber was designed and constructed specially for the setting of aorta perfusion (Fig. 1). All individual chamber and tubing parts were autoclaved or used as sterile disposables. Assembly was done under sterile conditions.

The rat was narcotized with intraperitoneal injection of pentobarbital ( $200 \mathrm{mg} / \mathrm{kg}$ body weight). After reaching surgical anesthesia, the abdomen was opened and the abdominal aorta was subse- 


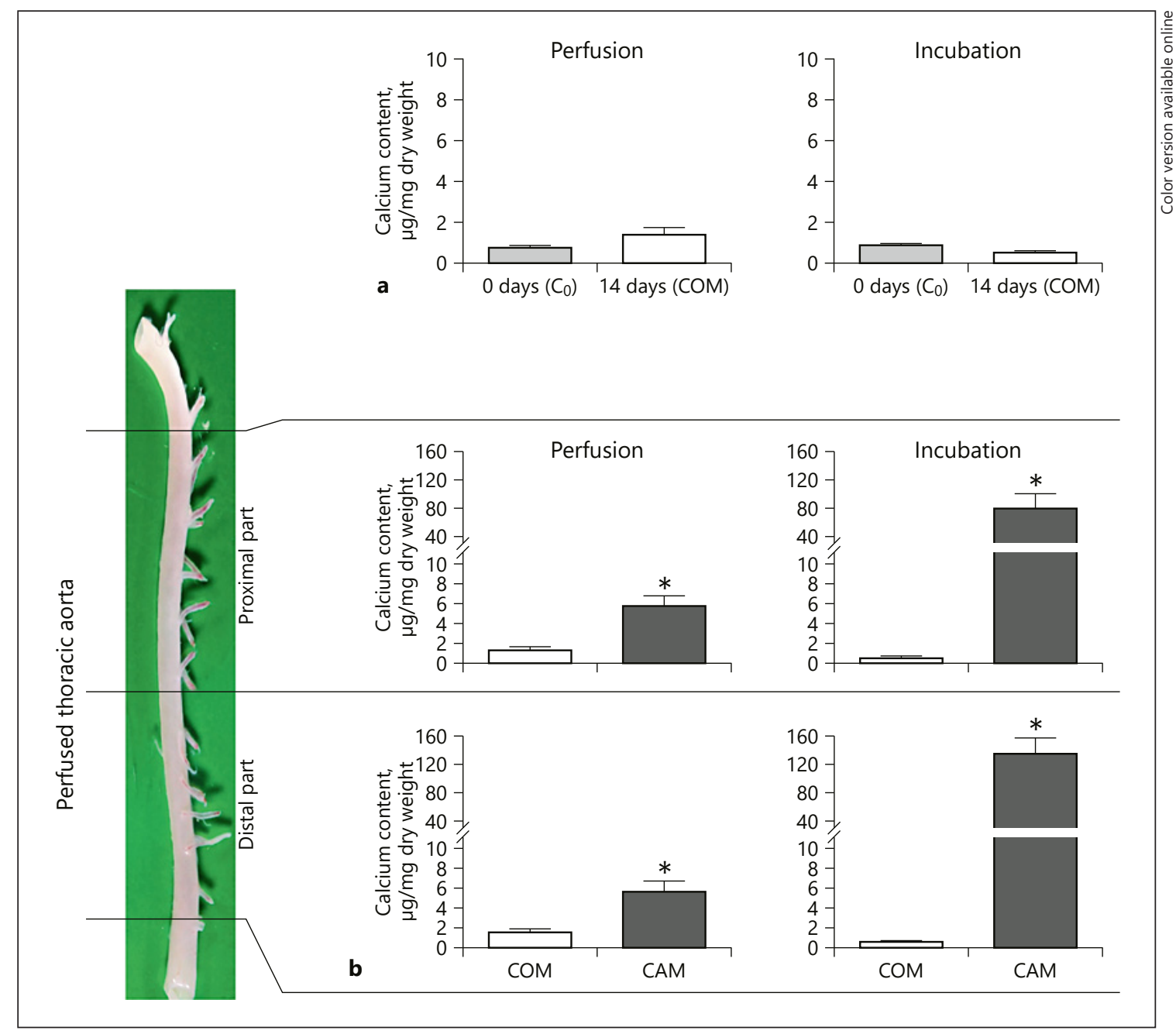

Fig. 2. Quantification of calcification. a Comparison of initial calcium content $\left(C_{0}\right)$ and the content after perfusion or incubation with COM. b Comparison of the calcium content in the proximal and distal aortic parts after perfusion and incubation with COM and CAM. $n=15,{ }^{*} p<0.05$, Mann-Whitney U test, two-tailed.

quently freed mechanically from the surrounding adipose tissue. Next, the thoracic cavity was opened and the aorta was dissected along the desired length. The soft tissue surrounding the aorta was grasped with forceps and the aorta gradually liberated together with the adventitia by cutting in the cranial direction through the soft tissue and the branches with a generous safety distance. For aortic explantation, the aorta was proximally cut close to the aortic valve of the heart and distally directly beneath the renal arteries. The aorta was transferred to a cell culture dish with phosphatebuffered saline. Peripheral fat tissue was carefully removed with micro scissors and forceps without impairment of aortic branches using a stereomicroscope. All outgoing vessels were cut and carefully closed using an electrocauter system (KLS Martin, Tuttlingen, Germany) with bipolar forceps. The explanted aorta was cut beneath the left subclavian artery and above the celiac artery. The thoracic aortic part between these cuts was used for perfusion. For aorta clamping, catheter systems (14-16 gauge, without a needle) were used.

The buffer in the chamber surrounding the aorta is composed of sodium chloride $(100 \mathrm{mmol} / \mathrm{L})$, potassium chloride $(5 \mathrm{mmol} / \mathrm{L})$, magnesium chloride $(0.5 \mathrm{mmol} / \mathrm{L})$, sodium dihydrogenphosphate $(0.5 \mathrm{mmol} / \mathrm{L})$, glucose $(10 \mathrm{mmol} / \mathrm{L})$, and $1 \%$ antibiotics (penicillin/ streptomycin). The chamber was completely filled with buffer. For aortic perfusion a control medium (COM) and a calcification medium (CAM) were used. The COM consisted of Dulbecco's modified eagle media ( $4.5 \mathrm{~g} / \mathrm{L}$ glucose) supplemented with $15 \%$ fetal calf sera and $1 \%$ penicillin/streptomycin. For the CAM, ascorbic acid $(284 \mu \mathrm{mol} / \mathrm{L})$ and sodium dihydrogenphosphate $(5 \mathrm{mmol} / \mathrm{L})$ were additionally added. In order to be able to compare the results obtained under perfusion conditions with those of previous and ongoing studies, the same CAM as in the previous published investigations was used $[5,6]$. All buffers and media were used in sterile 
conditions. The aorta was constantly perfused via a peristaltic pump with COM or CAM for 14 days by $37^{\circ} \mathrm{C}, 5 \%$ carbon dioxide, and humidity conditions of $95 \%$. The perfusion media was changed 4 times during the experiment. Simultaneously, up to 4 aortas were perfused with the same media per chamber experiment.

The chamber is closed with a lid. Gas exchange is facilitated to a sterile filter system. At the end of the perfusion experiment, the aorta was cut in proximal and distal subparts (Fig. 2) for further analysis (formalin conservation, cryoconservation, and decalcification). A proximal part and a distal part of the aorta were cut into rings and incubated separately (ex vivo without perfusion) as the control.

\section{Staining Methods}

Aortic rings were fixed with buffered formalin (4\%) for $24 \mathrm{~h}$. Afterwards, they were dehydrated and embedded in paraffin. Aortic slices $(4 \mu \mathrm{m})$ were stained with hematoxylin/eosin $(\mathrm{H} / \mathrm{E})$ and Alizarin Red. Each sample was stained as serial cutting of at least 3 aortic rings. Image acquisition and analysis were done using the AxioVert 200M System with Zen software (Zeiss, Jena, Germany).

\section{Calcium Content}

Aortic rings were decalcified with hydrochloric acid (0.6 $\mathrm{mol} / \mathrm{L}$ ). The calcium content was measured in technical duplicates using a colorimetric Calcium C assay kit (ScienCell, Berlin, Germany) according to the manufactures' protocol. The calcium content was normalized to its tissue dry weight.

\section{Gene Expression}

RNA was extracted from the cryoconserved aortic ring for COM- and CAM-perfused aorta as well as from the incubation experiment. The RNA extraction was done using Trizol ${ }^{\mathrm{TM}}$ as per the manufacturer's instructions. The amount and purity of RNA were determined by measuring the OD using a NanoDrop photometer (Thermo Fisher Scientific, Dreieich, Germany). RNA was reverse transcribed into cDNA using a High-Capacity cDNA Reverse Transcription $\mathrm{Kit}^{\mathrm{TM}}$ (Thermo Fisher Scientific). The mRNA expression of the investigated proteins was determined by quantitative real-time PCR using the CFX System with a SybrGreen kit (Bio-Rad, Munich, Germany). The oligonucleotide sequence is as follows: bone morphogenetic protein 2 (BMP-2) forward $5^{\prime}$-ACT TCC CGA CGC TTC TTC TTC A-3', reverse $5^{\prime}$-GGC CAC TTC CAC CAC AAA CC-3' [7], SM22 $\alpha$ forward 5'-AGA GGG GCC TCA CAG GCT GG-3', reverse $5^{\prime}$-ACA GCT GGG AAC AGG GGC CA-3', GAPD forward 5'-AGA CAG CCG CAT CTT CTT GT-3', reverse 5' ${ }^{\prime}$-CTT GCC GTG GGT AGA GTC AT- ${ }^{\prime}$. GAPD was ordered from Biozol (Eching, Germany) and all others were obtained from TibMolBiol (Berlin, Germany). Quantification of gene expression was normalized to GAPD as the house-keeping gene.

\section{Statistical Analysis}

Data are presented as means \pm SEM ( $N$ represents the number of aortas from different animals). The experiments were done in at least 3 independent experiments with 1-4 aortas per approach. Statistical analyses were performed by using GraphPad Prism (version 5) software. To evaluate differences between treatment groups, Anova and Mann-Whitney $U$ tests were applied. $p<0.05$ was considered statistically significant.

\section{Results and Discussion/Conclusion}

This technical note describes a novel ex vivo rat aortic perfusion system for vascular calcification studies. The induction of vascular medial calcification by standard ex vivo incubation of aortic rings was compared with the novel IPA method. High-phosphate medium was used in both settings; however, while aortic rings in the standard ex vivo method are incubated in the medium, the aortic tube in the IPA method is constantly endoluminally perfused with it. Both settings were employed for 14 days and the calcium content was measured thereafter. Corresponding aortic rings were decalcified directly after dissection $\left(\mathrm{C}_{0}\right)$ and the calcium content was quantified as a control.

\section{Calcification: Comparison of IPA Perfusion and ex vivo Incubation}

As known from previous studies, in vitro and ex vivo calcification of VSMC could be induced by using medium with a high phosphate content $[5,6,8]$. Therefore, CAM containing a high phosphate concentration and COM containing a physiological phosphate concentration were used in the following experiments.

Due to putative differences between the properties of different aortic segments [4], the proximal and distal thoracic tissues were investigated independently using COM and CAM. Incubation (without perfusion) with CAM induced a strong increase in calcium content (90- to 135fold) compared to incubation with COM in both investigated aortic parts. Previous studies of ex vivo calcification (without perfusion) have shown similar results [6], with differences in calcium content due to different vascular beds used and variations in stimulation procedure and time [9]. Using IPA, perfusion with CAM induced a significant increase in calcium content compared to perfusion with COM of the proximal and distal aorta, respectively (proximal: $\sim 5$-fold, distal: $\sim 6$-fold). Interestingly, the calcium content of CAM-perfused proximal and distal aortas was significantly lower compared with the CAM incubation procedure, whereas the effects of COM in both settings were comparable (Fig. 2b). The different exposure of VSMC to the high-phosphate medium might be one reason for the differences in calcium content. In the incubation procedure of aortic rings, the tissue and cells are exposed from all sites to the medium. In contrast, perfusion using the IPA method enables administration of the medium from the luminal site only. The endothelial barrier might play a relevant role in the reduced calcification process. Concerning internal controls, there was no sig- 
Fig. 3. mRNA gene expression mRNA expression of BMP-2 (a) and SM22a (b) detected by quantitative real-time PCR in aortic tissue after perfusion or incubation with CAM (black bar) or COM (white bar). $n \leq 5,{ }^{*} p<0.05$, Mann-Whitney U test, two-tailed.
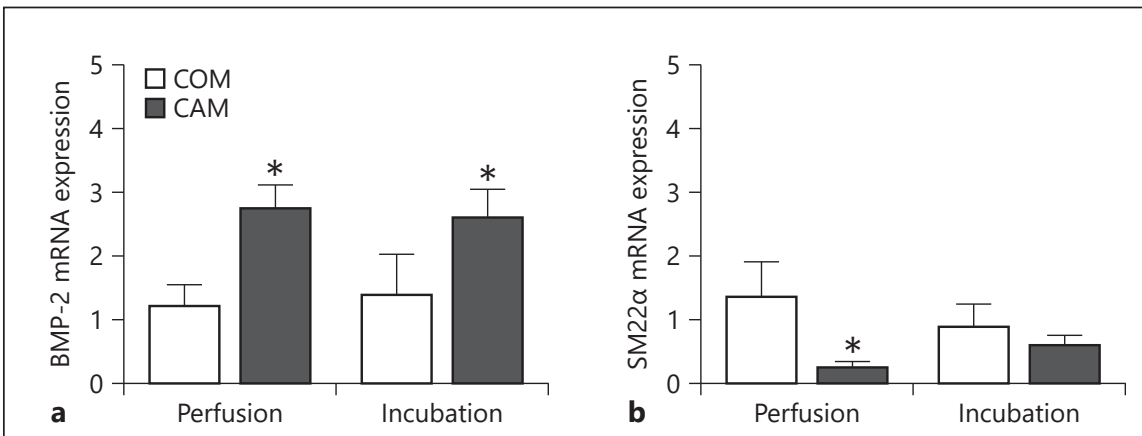

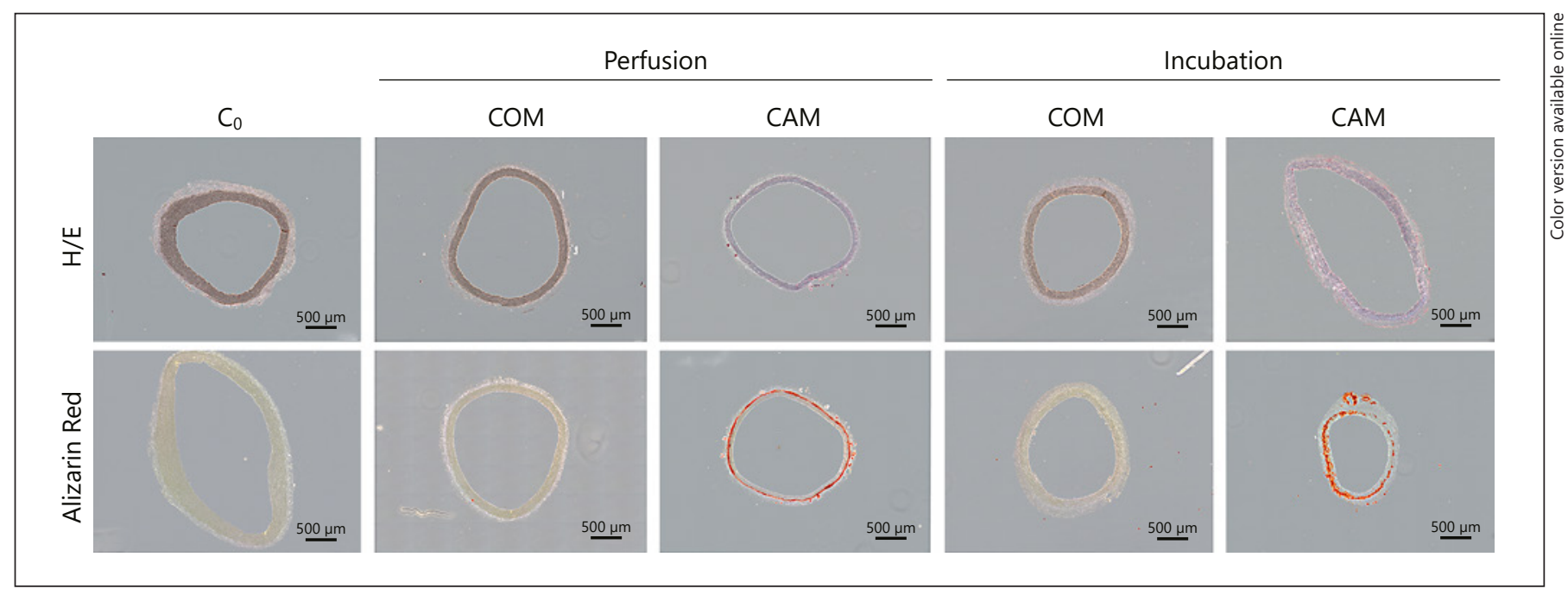

Fig. 4. Histological staining. $\mathrm{H} / \mathrm{E}$ and Alizarin Red staining of representative images.

nificant difference in calcium content observed between $\mathrm{COM}$ and $\mathrm{C}_{0}$ in the case of incubation and perfusion (Fig. 2a). Handling and the mere perfusion procedure did not induce calcification, because perfusion with COM did not significantly increase the aortic calcium content compared to ex vivo incubation without perfusion.

In addition, the mRNA gene expression of osteogenic BMP-2 and SM22 $\alpha$, a smooth muscle marker, was measured in the aortic tissue to examine a phenotypic conversion of VSMC upon CAM perfusion. The mRNA expression of BMP-2 was significantly elevated after aortic perfusion and incubation of CAM compared to the COM perfused/incubated aorta (Fig. 3a), while SM22a decreased (Fig. 3b). These results further suggest the induction of aortic mineralization upon CAM perfusion.

To obtain information about tissue integrity and the localization of calcification, aortic slices were stained with
H/E for structural analysis and with Alizarin Red to confirm vessel calcification. For all investigated conditions, staining with $\mathrm{H} / \mathrm{E}$ showed tissue integrity. In this study, neither necrotic lesions (e.g., lack of nucleus staining) nor homogenous cytoplasm colorability could be observed in histologic analysis. In control slides $\left(\mathrm{C}_{0}\right)$ as well as within the slides after perfusion or incubation with COM, Alizarin Red staining revealed no calcification within the vessel wall. In contrast, perfusion and incubation of the aortic tissue with CAM induced mineralization of the vessel wall (Fig. 4).

\section{IPA Compared to Other Known Calcification Studies}

Currently, different cell culture models (ex vivo or in vivo) exist, with pros and cons. In vitro cell culture models using VSMC represent the easiest model to study calcification. In this simplified experimental setting, inducers 
and inhibitors could be investigated; the calcium content, the gene expression profile, and the enzyme activity could be used as readout. However, the physiological tissue organization of the cells is lost in culture. Therefore, processes involving cooperative interaction with the extracellular matrix cannot be investigated. Studying ex vivo effects using aortic rings partly overcomes this problem. Here, a primary calcification in the media alongside the elastic laminae can be induced $[5,6,10,11]$. Nevertheless, it has to be considered, that the intima, the media, and the adventitia of the vessel wall are exposed to the test substances.

In vivo models represent a physiological but also the most complex experimental setting. Their advantage is that transgenic and knockout animals can be investigated. However, rodents are resistant to the development of medial calcification. For the induction of vascular calcification, the homeostasis of the animal must be altered severely, e.g., by the induction of chronic kidney disease, hyperparathyroidism, or hyperphosphatemia [12, 13], leading to an unpredictable alteration of signal pathways. Therefore, the transmission of results from these studies is limited.

The new ex vivo model of the IPA described here might fill this gap between ex vivo studies using aortic rings and in vivo animal models. With this new method, it is possible to investigate aortic tissue in a physiological manner via stimulation from the luminal site using perfusion for up to several weeks. In addition, it follows the $3 \mathrm{R}$ principle [14] for reduction of the medical conditions and pain of laboratory animals. The experimental models already established are designed to perfuse an aorta for up to hours and for visualization of processes via microscopy [15-17]. The IPA is designed for long-term perfusion for up to several weeks, allowing the study of medial vascular calcification. The main advantage of the IPA model is that the substances being tested could be administered specifically from the luminal site and therefore in a more physiological manner. This might be one explanation for the lesser but putatively more physiological calcification within the same time span compared to ex vivo incubation of aortic rings. A direct comparison of aortic calcium contents with in vivo models is difficult due to the complexity of and differences in the animal models. For example, vitamin $\mathrm{D}_{3}$-induced vascular calcification results in an about 10 -fold increase in calcium content [9], whereas induction of chronic kidney insufficiency results in an up to 100 -fold increase in a rat model [18].

\section{Study Limitations}

There are some limitations of the current study. First, solely staining methods checked the tissue viability. In the future, cell viability and functional integrity especially of the endothelial barrier will be measured via small vessel myography before and after perfusion of the organ for 14 days. Second, only one perfusion duration was analyzed. For better comparability to previous ex vivo experiments, 14 days were chosen. Third, only aortas from 2 -month-old male rats were investigated. As known from the literature, age and sex may influence the extent of vascular calcification. In the framework of the proofof-concept study, defined conditions were chosen to assess the effects on vascular calcification. In further studies, the gender-specific aspect of vascular calcification should be investigated with this new technique. Fourth, the wall shear stress induced by the aortic perfusion could not be measured directly. However, we measured the perfusion pressure using a transducer in preliminary experiments. Furthermore, a peristaltic perfusion mode was chosen with a defined mean perfusion pressure and frequency. Therefore, parameters determining the shear stress were standardized. Fifth, the current study investigated the effect of different perfusion media on vascular calcification in healthy rat aorta. The effects of different perfusion pressures and comorbidities, e.g., chronic kidney disease, are highly interesting and will be investigated in further studies. Sixth, the perfusion of mouse aorta using this novel technique will broaden the experimental spectrum.

\section{Novelty and Conclusion}

The current study summarizes the experimental procedure and first proof-of-concept for vascular calcification analysis in an ex vivo perfused aorta. For the first time, this model has enabled ex vivo perfusion of the aorta, allowing studies with inducers/inhibitors of vessel calcification under controlled conditions via perfusion from the endoluminal site. A detailed analysis of the processes of vascular calcification with histological and biochemical methods could be performed after long-term aortic perfusion. It enables investigation of pharmacological drugs in calcification process and studying the role of the endothelium. Furthermore, this method could be used for other vascular beds of different species. More studies are needed regarding, e.g., different incubation times and determination of perfusion pressure before routine use. 


\section{Acknowledgement}

The authors are deeply grateful to Dieter Bräuer who assembled the perfusion chamber according to our requirements. We thank Brigitte Egbers, Katharina Kuschfeldt, and Nadine Neitzel for their excellent technical assistance and Jaqueline Herrmann for her support with image acquisition.

\section{Statement of Ethics}

The animal experiments were in conformance with internationally accepted standards and the appropriate institutional review body approved them.

\section{Disclosure Statement}

The authors have no conflict of interests to declare.

\section{Funding Sources}

This research did not receive any specific grant from funding agencies in the public, commercial, or not-for-profit sector.

\section{Author Contributions}

M.S., M.T., and M.G. are responsible for the conception and design of this study and the perfusion chamber. M.S., M.T., M.G., and W.Z. wrote and drafted this article. M.S., M.T., N.V.S., and M.B. performed the experiments and analyzed and interpreted the results. R.L. and A.R. importantly contributed to the explant and coagulation technique of the tissue preparation. All of the authors read an approved the final version of this paper and critically revised it for important intellectual content.

\section{References}

1 Tölle M, Reshetnik A, Schuchardt M, Höhne $M$, van der Giet M. Arteriosclerosis and vascular calcification: causes, clinical assessment and therapy. Eur J Clin Invest. 2015 Sep;45(9): 976-85.

2 Neven E, De Schutter TM, De Broe ME, D'Haese PC. Cell biological and physicochemical aspects of arterial calcification. Kidney Int. 2011 Jun;79(11):1166-77.

3 Leopold JA. Vascular calcification: mechanisms of vascular smooth muscle cell calcification. Trends Cardiovasc Med. 2015 May; 25(4):267-74.

4 Schlieper G. Vascular calcification in chronic kidney disease: not all arteries are created equal. Kidney Int. 2014 Mar;85(3):501-3.

5 Schuchardt M, Tölle M, Prüfer J, Prüfer N, Huang T, Jankowski V, et al. Uridine adenosine tetraphosphate activation of the purinergic receptor P2Y enhances in vitro vascular calcification. Kidney Int. 2012 Feb;81(3):25665.

6 Prüfer J, Schuchardt M, Tölle M, Prüfer N, Höhne M, Zidek W, et al. Harmful effects of the azathioprine metabolite 6-mercaptopurine in vascular cells: induction of mineralization. PLoS One. 2014 Jul;9(7):e101709.
7 Maciel TT, Melo RS, Campos AH. The bone morphogenetic protein antagonist gremlin promotes vascular smooth muscle cell apoptosis. J Vasc Res. 2009;46(4):325-32.

8 Mori K, Shioi A, Jono S, Nishizawa Y, Morii $\mathrm{H}$. Dexamethasone enhances In vitro vascular calcification by promoting osteoblastic differentiation of vascular smooth muscle cells. Arterioscler Thromb Vasc Biol. 1999 Sep;19(9): 2112-8.

9 Ha CM, Park S, Choi YK, Jeong JY, Oh CJ, Bae $\mathrm{KH}$, et al. Activation of Nrf2 by dimethyl fumarate improves vascular calcification. Vascul Pharmacol. 2014 Oct;63(1):29-36.

10 Lomashvili KA, Cobbs S, Hennigar RA, Hardcastle KI, O’Neill WC. Phosphate-induced vascular calcification: role of pyrophosphate and osteopontin. J Am Soc Nephrol. 2004 Jun; 15(6):1392-401.

11 Deng L, Huang L, Sun Y, Heath JM, Wu H, Chen Y. Inhibition of FOXO1/3 promotes vascular calcification. Arterioscler Thromb Vasc Biol. 2015 Jan;35(1):175-83.

12 Shobeiri N, Adams MA, Holden RM. Vascular calcification in animal models of CKD: A review. Am J Nephrol. 2010;31(6):471-81.
13 Neven E, D'Haese PC. Vascular calcification in chronic renal failure: what have we learned from animal studies? Circ Res. 2011 Jan; 108(2):249-64.

14 Russell WM, Bruch RL. The principles of human experimental technique. London: Methuen; 1959.

15 Megens RT, Reitsma S, Schiffers PH, Hilgers RH, De Mey JG, Slaaf DW, et al. Two-photon microscopy of vital murine elastic and muscular arteries. Combined structural and functional imaging with subcellular resolution. J Vasc Res. 2007;44(2):87-98.

16 Wang X, Wolf MP, Keel RB, Lehner R, Hunziker PR. Polydimethylsiloxane embedded mouse aorta ex vivo perfusion model: proofof-concept study focusing on atherosclerosis. J Biomed Opt. 2012 Jul;17(7):076006.

17 van Zandvoort M, Engels W, Douma K, Beckers L, Oude Egbrink M, Daemen M, et al. Two-photon microscopy for imaging of the (atherosclerotic) vascular wall: a proof of concept study. J Vasc Res. 2004 Jan-Feb;41(1): 54-63.

18 Shobeiri N, Pang J, Adams MA, Holden RM Cardiovascular disease in an adenine-induced model of chronic kidney disease: the temporal link between vascular calcification and haemodynamic consequences. J Hypertens. 2013 Jan;31(1):160-8. 